\begin{tabular}{|c|l|}
\hline Title & Exciton spin relaxation in GaN observed by spin grating experiment \\
\hline Author(s) & Ishiguro, T.; Toda, Y.; A dachi, S. \\
\hline Citation & $\begin{array}{l}\text { A pplied Physics Letters, 90(1), 011904 } \\
\text { https://doi.org/L0.1063/2430402 }\end{array}$ \\
\hline Issue Date & 2007-01-01 \\
\hline Doc URL & http://hdl.handle.net/2115/17233 \\
\hline Rights & ○ 2007 A merican Institute of Physics \\
\hline Type & article \\
\hline File Information & APL90-1.pdf \\
\hline
\end{tabular}

Instructions for use 


\title{
Exciton spin relaxation in GaN observed by spin grating experiment
}

\author{
T. Ishiguro, Y. Toda, ${ }^{a}$ and S. Adachi \\ Department of Applied Physics, Hokkaido University, Kita 13 Nishi 8 Kita-ku, Sapporo 060-8628, Japan
}

(Received 16 June 2006; accepted 4 December 2006; published online 3 January 2007)

\begin{abstract}
The authors studied the exciton spin relaxation of bulk GaN by creating spin polarization gratings using degenerate four-wave mixing spectroscopy. The spectrally resolved analysis achieved with this technique facilitated the direct evaluation of spin polarizations in the individual excitons $(A$ and $B$ excitons). The spin polarizations for each exciton decay very quickly $\left(\tau_{s} \sim 1 \mathrm{ps}\right)$ at low temperatures. Moreover the $\tau_{s}$ is faster than the dephasing time $T_{2}$ throughout the measured temperature range, suggesting the existence of fast intrinsic spin relaxation processes, which can be attributed to a large exchange constant characterized in GaN. (C) 2007 American Institute of Physics.

[DOI: $10.1063 / 1.2430402]$
\end{abstract}

Because of the technological importance of spin-based electronics, so-called spintronics, ${ }^{1}$ considerable effort has recently been devoted to understanding the optically induced spin polarization in $\mathrm{GaN}$ and related alloys, both experimentally $^{2-5}$ and theoretically. ${ }^{6-9}$ A noteworthy issue is the significant variation in the spin relaxation time obtained in previous experiments; in Ref. 2, a long electron spin coherence of up to $20 \mathrm{~ns}$ was observed in Si-doped GaN by using time-resolved Faraday rotation measurements, while short (subpicosecond) excitonic-spin relaxation times were observed in a $\mathrm{GaN}$ epilayer by using spin-dependent transient reflectivity change measurements. ${ }^{3}$ Although theoretical work has suggested a significant influence of the sample quality on the spin dynamics in semiconductors, ${ }^{7}$ the mechanism that causes the difference between these two results is still unclear.

In this work, we studied exciton spin relaxation in bulk GaN by using spectrally resolved four-wave mixing (SRFWM) measurements. Strikingly fast spin relaxations, which were even faster than the dephasing times $T_{2}$, were observed for each exciton resonance at low temperatures. The relaxation time in an $A$ exciton $\left(X_{A}\right)$ decreases with increasing temperature, and then reaches the experimental resolution limit above $100 \mathrm{~K}$. The results can be understood in terms of an excitonic effect in $\mathrm{GaN}$.

Figure 1(a) is a schematic illustration of the experiment. In order to evaluate the spin lifetime in the spectral domain, we employed a three-pulse FWM measurement. ${ }^{10-12} \mathrm{~A}$ frequency doubled, mode-locked Ti:sapphire laser which produced a pulse width of $\sim 200 \mathrm{fs}$ (spectral width of $\sim 13 \mathrm{meV}$ ) at a repetition rate of $76 \mathrm{MHz}$ was employed as an excitation light source. Two simultaneous excitation pulses and one probe pulse with a controlled time delay were focused at the same point on the sample surface using a lens $(f=200 \mathrm{~mm})$. These three pulses have almost the same power of about $0.3 \mathrm{~mW}$. The pump pair consisting of crosslinear (colinear) polarizations of wave vectors $\mathbf{k}_{1}$ and $\mathbf{k}_{2}$ forms an exciton spin polarization (population) grating [Fig. 1(b)] which mainly decays via the exciton spin relaxation (recombination) process. ${ }^{13}$ A third probe pulse of wave vector $\mathbf{k}_{3}$ diffracts off this grating to produce the FWM sig-

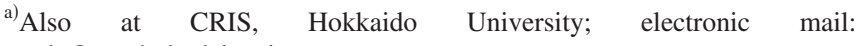
toda@eng.hokudai.ac.jp
}

nal in the $\mathbf{k}_{4}=\mathbf{k}_{3} \mp \mathbf{k}_{2} \pm \mathbf{k}_{1}$ direction, which was selected spatially by an iris and resolved spectrally by a $30-\mathrm{cm}-$ long monochromator with a charge coupled device detector. The spectral resolution is $0.7 \mathrm{meV}$.

Here we briefly describe the excitonic-spin polarization in wurtzite GaN. Figure 1(c) shows an overview of the energy structure of an electron and holes for the $A$ and $B$ excitons $\left(X_{A}\right.$ and $\left.X_{B}\right)$ in GaN based on the general group theory. The direct products of the lowest conduction band with $\Gamma_{7}$ symmetry and the topmost of the valence band with $\Gamma_{9}$ give $X_{A}$ with $\Gamma_{5}(x, y)$ and $\Gamma_{6}$ symmetry, where only $\Gamma_{5}(x, y)$ is optically allowed. On the other hand, $X_{B}$ arises from the second valence band with $\Gamma_{7}$ symmetry and consists of two optically allowed states $\Gamma_{1}(z)$ and $\Gamma_{5}(x, y)$. Both in $X_{A}$ and $X_{B}, \Gamma_{5}(x, y)$ is decomposed into two opposite circularly polarized spin states: $|+1\rangle$ and $|-1\rangle$.

In our study, we investigated a $70-\mu \mathrm{m}$-thick freestanding $\mathrm{GaN}$ sample, which was grown by the two-flow metalorganic chemical vapor deposition method using the lateral

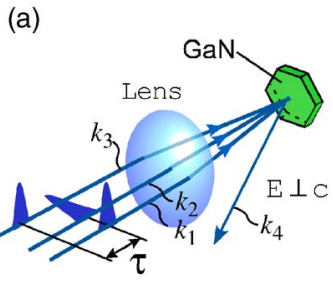

(c) (b)

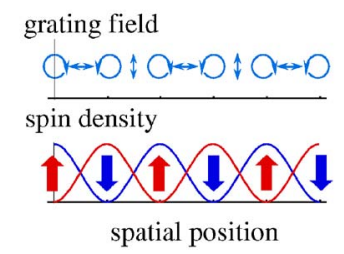

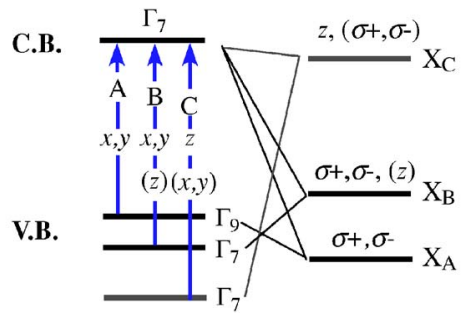

FIG. 1. (Color online) (a) Excitation scheme for generating a spin polarization grating induced by cross-linearly polarized pump pulses with wave vectors $\mathbf{k}_{1}$ and $\mathbf{k}_{2}$. The pump pulses were mechanically delayed with respect to the third probe pulse with wave vector $\mathbf{k}_{3}$. (b) Spatial distribution of polarization in the cross-polarized excitation (upper) and corresponding spin density distribution (lower). (c) Schematic of energy bands at the $\Gamma$ point and the internal structure of excitons in wurtzite crystals based on the general group theory. 

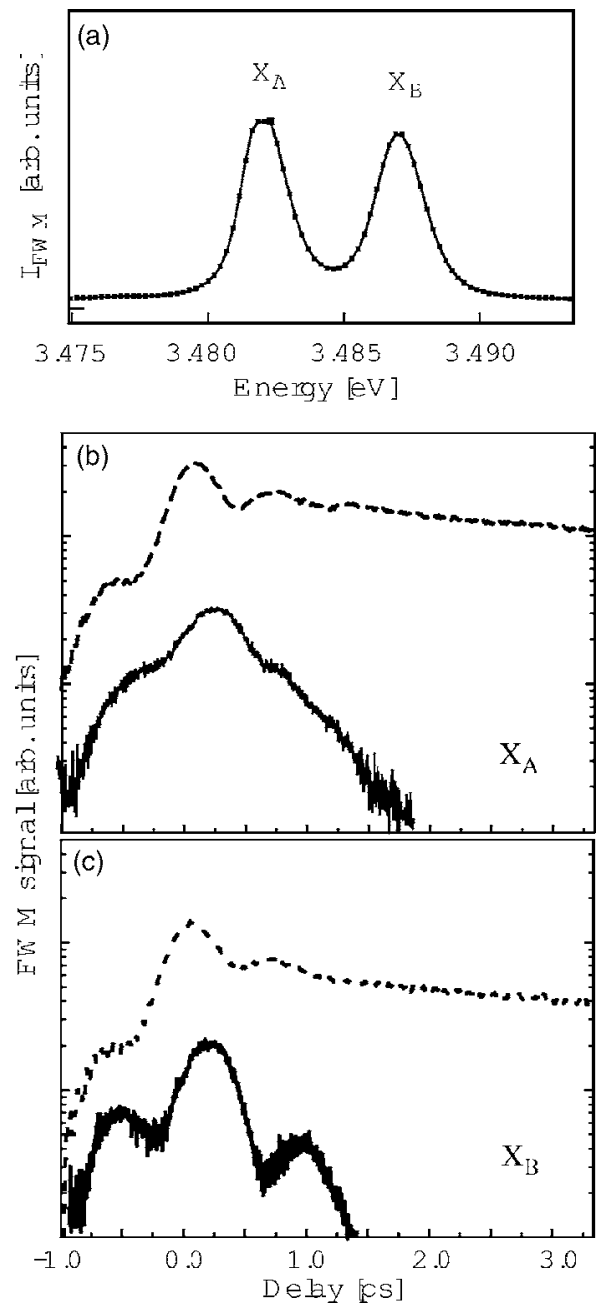

FIG. 2. (a) Typical FWM spectrum of the sample at delay time $\tau=0 \mathrm{~s}$. The solid line is a Lorentzian fitting. SRFWM for (b) $X_{A}$ and (c) $X_{B}$. The solid and dashed lines represent the spin polarization transient and the population transient, respectively. The results were obtained at $10 \mathrm{~K}$.

epitaxial overgrowth technique. We should point out that the residual carrier concentration was of the order of $10^{14} \mathrm{~cm}^{-3}$, indicating a small quantity of residual impurities. The sample characteristics are described in detail in Ref. 14.

A typical SRFWM spectrum at zero time delay is shown in Fig. 2(a), which was obtained for the pump pulses in a cross-linearly polarized configuration. In the spectrum, the lower- and higher-energy peaks can be identified as $X_{A}$ and $X_{B}$, respectively. The exciton energies obtained from the Lorentzian fitting to the data were 3.4809 and $3.4862 \mathrm{eV}$, respectively. There was an energy separation of $\sim 5 \mathrm{meV}$ between the two excitons. This separation is smaller than that of the excitation laser spectrum and so means that it is impossible to achieve the selective excitation of individual exciton levels that is required if we are to obtain spindependent transient measurements. ${ }^{3,4}$ In the two-pulse FWM measurements, the $T_{2}$ values of each exciton, where we assume the homogeneous broadening, are estimated to be 1.6 and $1.7 \mathrm{ps}$, respectively. These values are consistent with the linewidths in the spectrum. ${ }^{15}$ This consistency and the relatively long $T_{2}$ account for the high crystal quality of our thick sample.

Figures 2(b) and 2(c) display the results of three-pulse SRFWM for $X_{A}$ and $X_{B}$, respectively, at the lowest temperature $(T=10 \mathrm{~K})$. An intense signal with a long decay was Downloaded 08 Feb 2007 to 133.87.26.100. Redistribution subjec
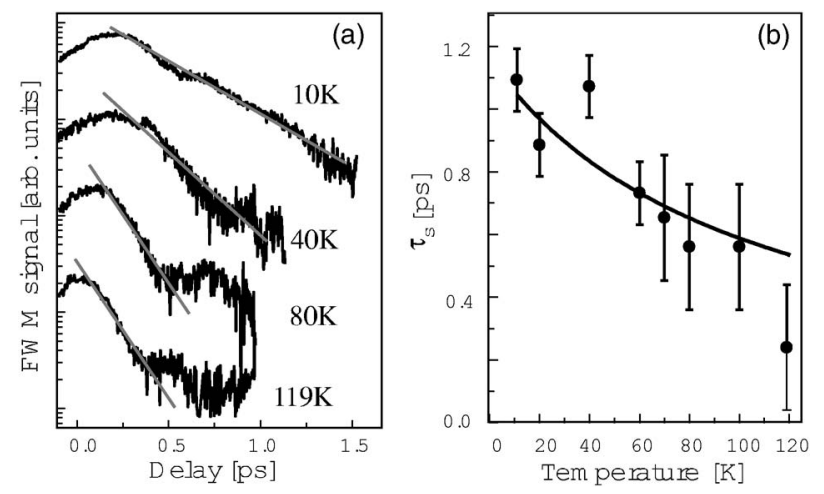

FIG. 3. (a) Spin polarization transients of $X_{A}$ at various temperatures. (b) Plots of decay time obtained from a least-square fit to the data. The solid curve is the result of fitting using a model described in the text.

observed by using colinearly polarized pump excitation, in which the fast decay component corresponds to the dephasing process, while the subsequent slow decay corresponds to a lifetime of the order of several tens of picoseconds. In contrast, the FWM signal observed in the cross-linear configuration shows a very rapid decay. With this geometry, the signal reflects the decay of the periodic pattern of the polarization grating [Fig. 1(b)]. A small oscillatory component superimposed on the exponential decay originates from the remnant of the two-pulse FWM signals (e.g., $2 \mathbf{k}_{2}-\mathbf{k}_{1}$ ) after spatial filtering, and we disregard this contribution when evaluating the decay. By fitting $\exp \left(-2 \tau / \tau_{s}\right)$ to the data, we obtained a typical spin relaxation time of $\tau_{s}=1.2 \mathrm{ps}$ at $X_{A}$ resonance at $10 \mathrm{~K}$. On the other hand, the oscillation is dominant at $X_{B}$, indicating a small amount of spin polarization. The reduced polarization compared with $X_{A}$ is due to the excited state nature of $X_{B}$, which provides an additional relaxation channel toward the lowest $X_{A}$ state.

Figure 3 (a) shows semilog plots of the SRFWM intensities of $X_{A}$ at various temperatures. The pump polarizations were set so that they crossed. In Fig. 3(b), we plot $\tau_{s}$ obtained by a single exponential fit to the data. The $\tau_{s}$ value decreases monotonically with increasing temperature, and finally becomes comparable to the pulse duration above $T \sim 100 \mathrm{~K}$. This temperature dependence of $\tau_{s}$ clearly indicates the scattering process contribution, which can be evaluated by comparison with the temperature dependence of $T_{2}$. For this purpose, we employ the fitting function expressed as $\tau_{s}(T)=2 \hbar /\left(\alpha_{0}+\beta_{\mathrm{ph}} T\right)$, where $\alpha_{0}$ and $\beta_{\mathrm{ph}}$ correspond to the homogeneous broadening $\Gamma_{h}$ at zero temperature for $T_{2}=2 \hbar / \Gamma_{h}$ and to the exciton-acoustic phonon interaction coefficient, respectively. From a least-square fitting procedure, we have obtained the values $\alpha_{0}=1.1 \mathrm{meV}$ and $\beta_{\mathrm{ph}}=11 \mu \mathrm{eV} / K$. The $\beta_{\mathrm{ph}}$ value is comparable to that for $T_{2}$ in $\mathrm{GaN}$ reported by several authors, ${ }^{16}$ while $\alpha_{0}$ is very large, meaning that $\tau_{s}$ is faster than $T_{2}$ at zero temperature. These results suggest that there must be intrinsic fast relaxation processes additional to the exciton-phonon-scatteringinduced relaxation. This will be discussed below.

Similar fast spin relaxation with a $\tau_{s}$ of $0.47-0.25 \mathrm{ps}$ at $150-225 \mathrm{~K}$ has been observed in a GaN epilayer by using spin-dependent transient reflectivity change measurements. ${ }^{3}$ In contrast, in Ref. 2, the electron spin coherence in $n$-doped $\mathrm{GaN}$ becomes as long as $\sim 20 \mathrm{~ns}$ at $5 \mathrm{~K}$. This noticeable difference might result from the relatively low quality of the former sample since defects and dislocations originating
to AIP license or copyright, see http://apl.aip.org/apl/copyright.jsp 
from the substrate will not relax in such a thin sample. Although GaN essentially has a high defect density, the observed subpicosecond spin relaxation time in our thick sample supports the generality of a fast $\tau_{s}$ in terms of excitonic transitions, which in turn indicates that the difference in spin relaxation time can be attributed to the difference between electron spin and exciton spin relaxations.

Usually, electron/hole spin is affected by a spin-orbit (SO) interaction: ${ }^{17}$ a strong SO coupling results in a large electron spin splitting at large $\mathbf{k}$, where scattering caused by impurities and phonons gives rise to efficient spin relaxation. Since GaN exhibits a small SO splitting $\Delta_{\mathrm{SO}} \approx 13 \mathrm{meV}$, which is smaller than $\mathrm{GaAs}\left(\Delta_{\mathrm{SO}} \approx 350 \mathrm{meV}\right)$, a long-lived electron spin is predicted ${ }^{8}$ and accounts well for the electron spin experiments on doped $\mathrm{GaN}^{2}$ On the other hand, in an electron-hole picture, excitonic exchange interactions provide an additional spin relaxation channel that accounts for the simultaneous flip between electrons and holes, known as the Bir-Aronov-Pikus mechanism. ${ }^{18}$ Optical experiments reveal a large spin exchange constant $\gamma=0.6 \mathrm{meV}$ of GaN, ${ }^{19-21}$ which is about three times that of GaAs $(\gamma=0.25 \mathrm{meV})$. Since $\tau_{s}$ is inversely proportional to the square of the exchange energy, we can expect an order of magnitude reduction in $\tau_{s}{ }^{22}$ Moreover, strikingly fast hole spin relaxation was theoretically predicted in $\mathrm{GaN}$ due to the weak SO coupling and the nearly degenerate large effective mass. ${ }^{8}$ In this case, the exciton spin relaxation time shortens as a result of the large exchange interaction.

It is important to note that $\tau_{s}$ is even faster than $T_{2}$ evaluated by two-pulse FWM for temperatures up to around $120 \mathrm{~K}$. Moreover, the $\beta_{\mathrm{ph}}$ value for $\tau_{s}$ is nearly identical to that for $T_{2}$, indicating that the contribution of motional narrowing is small. The fact that $\tau_{s}$ is faster than $T_{2}$ indicates that scattering-induced relaxations are not dominant for $\tau_{s}$ although they provide a temperature dependent contribution. As a consequence, we conclude that the fast $\tau_{s}$ is determined by the intrinsic spin-flip processes of $\mathrm{GaN}$ excitons, which are possibly caused by the large exchange interaction.

In summary, we have investigated the three-pulse FWM response of exciton spin polarization in a high-quality bulk $\mathrm{GaN}$. The spin relaxation times in each of the exciton states were estimated to be $\sim 1 \mathrm{ps}$ at $10 \mathrm{~K}$. Based on a disagreement from long-lived electron spin, we argue that the short spin relaxation times observed at exciton resonance originate from the excitonic interaction. A large exchange interaction in $\mathrm{GaN}$ provides a plausible explanation for this efficient spin relaxation.

The authors are grateful to T. Mukai (Nichia Corp.) for preparing a high-quality GaN sample.

${ }^{1}$ Semiconductor Spintronics and Quantum Computation, edited by D. D. Awschalom, N. Samarth, and D. Loss (Springer, Berlin, 2002).

${ }^{2}$ B. Beschoten, E. Johnston-Halperin, D. K. Young, M. Poggio, J. E. Grimaldi, S. Keller, S. P. DenBaars, U. K. Mishra, E. L. Hu, and D. D. Awschalom, Phys. Rev. B 63, 121202 (2001).

${ }^{3}$ T. Kuroda, T. Yabushita, T. Kosuge, A. Tackeuchi, K. Taniguchi, T. Chinone, and N. Horio, Appl. Phys. Lett. 85, 3116 (2004).

${ }^{4}$ S. Nagahara, M. Arita, and Y. Arakawa, Appl. Phys. Lett. 86, 242103 (2005).

${ }^{5}$ C. Y. Hu, K. Morita, H. Sanada, S. Matsuzaka, Y. Ohno, and H. Ohno, Phys. Rev. B 72, 121203(R) (2005).

${ }^{6}$ D. Jena, Phys. Rev. B 70, 245203 (2004).

${ }^{7}$ S. Krishnamurthy, M. V. Schilfgaarde, and N. Newman, Appl. Phys. Lett. 83, 1761 (2003).

${ }^{8}$ Z. G. Yu, S. Krishnamurthy, M. V. Schilfgaarde, and N. Newman, Phys. Rev. B 71, 245312 (2005).

${ }^{9}$ P. H. Song and K. W. Kim, Phys. Rev. B 66, 035207 (2002).

${ }^{10}$ A. R. Cameron, P. Riblet, and A. Miller, Phys. Rev. Lett. 76, 4793 (1996).

${ }^{11}$ O. Ikeuchi, S. Adachi, H. Sasakura, and S. Muto, J. Appl. Phys. 93, 9634 (2003).

${ }^{12}$ S. Adachi, Y. Takagi, J. Takeda, and K. A. Nelson, Opt. Commun. 174, 291 (2000).

${ }^{13}$ Since the diffracted signals are proportional to the depth of the generated grating, exciton (spin) diffusion contributes to the signal decay as well as the recombination (spin relaxation) process. In these experiments, the grating pitch is so large that the diffusion effect cannot affect the grating decay on the time scale of interest.

${ }^{14}$ K. Torii, T. Deguchi, T. Sota, K. Suzuki, S. Chichibu, and S. Nakamura, Phys. Rev. B 60, 4723 (1999); T. Deguchi, D. Ichiryu, K. Toshikawa, K. Sekiguchi, T. Sota, R. Matsu, T. Azuhata, M. Yamaguchi, T. Yagi, S. Chichibu, and S. Nakamura, J. Appl. Phys. 86, 1860 (1999).

${ }^{15}$ Since the resonance linewidth of $\sim 2 \mathrm{meV}$ is only 2.5 times that for a homogeneous case (linewidth of $0.8 \mathrm{meV}$ from $T_{2} \sim 1.6 \mathrm{ps}$ ), the system is close to exhibiting homogeneous broadening.

${ }^{16}$ K. Hazu, A. Shikanai, T. Sota, K. Suzuki, S. Adachi, S. F. Chichibu, and T. Mukai, Phys. Rev. B 65, 195202 (2002); S. Pau, J. Kuhl, F. Scholz, V. Haerle, M. A. Khan, and C. J. Sun, Appl. Phys. Lett. 72, 557 (1998); A. J. Fischer, W. Shan, G. H. Park, J. J. Song, D. S. Kim, D. S. Yee, R. Horning, and B. Goldenberg, Phys. Rev. B 56, 1077 (1997).

${ }^{17}$ R. J. Elliott, Phys. Rev. 96, 266 (1954).

${ }^{18}$ G. L. Bir, A. G. Aronov, and G. E. Pikus, Zh. Eksp. Teor. Fiz. 69, 1382 (1975) [Sov. Phys. JETP 42, 705 (1976)].

${ }^{19}$ M. Julier, J. Campo, B. Gil, J. P. Lascaray, and S. Nakamura, Phys. Rev. B 57, R6791 (1998).

${ }^{20}$ P. P. Paskov, T. Paskova, P. O. Holtz, and B. Monemar, Phys. Status Solidi A 190, 75 (2002).

${ }^{21}$ Y. Toda, S. Adachi, Y. Abe, K. Hoshino, and Y. Arakawa, Phys. Rev. B 71, 195315 (2005).

${ }^{22}$ M. Z. Maialle, Phys. Rev. B 54, 1967 (1996). 